\title{
Inherent Feature Extraction and Soft Margin Decision Boundary Optimization Technique for Hyperspectral Crop Classification
}

\author{
Mr. M.C.Girish Babu, Dr. Padma M.C \\ Department of Computer Science and Engineering \\ PES College of Engineering \\ Mandya, India
}

\begin{abstract}
Crop productivity and disaster management can be enhanced by employing hyperspectral images. Hyperspectral imaging is widely utilized in identifying and classifying objects on the ground surface for various agriculture application uses such as crop mapping, flood management, identifying crops damaged due to flood/drought, etc. Hyperspectral imaging-based crop classification is a very challenging task because of spectral dimensions and poor spatial feature representation. Designing efficient feature extraction and dimension reduction techniques can address high dimensionality problems. Nonetheless, achieving good classification accuracies with minimal computation overhead is a challenging task in Hyperspectral imaging-based crop classification. In meeting research challenges, this work presents Hyperspectral imaging-based crop classification using soft-margin decision boundary optimization (SMDBO) based Support Vector Machine (SVM) along with Image Fusion-Recursive Filter (IFRF) and Inherent Feature Extraction (IFE). In this work, IFRF is used for reducing spectral features with meaningful representation. Then, IFE is used for differentiating physical properties and shading elements of different objects spatially. Both spectral and spatial features extracted are trained using SMDBO-SVM for performing hyperspectral object classification. Using SMDBO-SVM for Hyperspectral object classification aid in addressing class imbalance issues; thus, the proposed IFE-SMDBO-SVM model achieves better accuracies and with minimal misclassification in comparison with state-of-art statistical and Deep Learning (DL) based Hyperspectral object classification model using standard dataset Indian Pines and Pavia University.
\end{abstract}

Keywords-Crop classification; decision boundary; deep learning; dimensionality; feature selection; hyperspectral image; support vector machines

\section{INTRODUCTION}

Agriculture plays a very important role in improving major developing country economy. High productivity of food yield will aid in meeting food security. However, with global warming, it is hard to achieve a very high yield. A significant amount of crops is lost worldwide due to natural disasters such as drought, cyclones, and floods; leading to loss of life of farmers/people. A farmer requires timely relief of funds for disaster management. Allocating the right kind of funds is challenging, as farmer grows multiple crops within the same region. Thus, efficient crop identification methodologies are needed. Hyperspectral Imaging (HSI) is an efficient method used for crop identification. Extensive work has been done in recent times for crop recognition in agriculture environment such as Locally Adaptive Dictionary (LAD) through Multiscale Joint Collaborative Feature (MJCF) [1], spatial-spectral feature extraction through end-to-end deep learning framework [2], neural network learning framework for extracting adaptive Spatial-Spectral Features [3], Improved CNN framework combining Markov random fields for extracting spatial-spectral feature [4], Conditional Random Field and Deep Metric Learning for HSI classification [5]. However, following challenges such as high dimension size, presence of noise, and high similarity among spectral features, shapes, textures of different crops must be addressed in building an effective hyperspectral imaging-based crop classification method. Hyperspectral imaging consists of hundreds of Narrow Bands that are continuous with high spectral correlation. Thus, results in Hughes phenomenon, space, and computation complexity as shown in following work such as band selection through Endto-End deep learning architecture [7], hierarchical spatialspectral feature maps through CNN [8], spectral-spatial feature extraction using CNN and information measure [9], and Active learning-based CNN model [10], a hybrid model combining Inception and Deep Residual Network [11] for HSI crop classification.

The crop classification accuracies can be improved by the utilization of feature extraction and feature selection methods. Existing methodologies predominantly used Principal Component Analysis (PCA) and Independent Component Analysis (ICA) for reducing the feature size of hyperspectral images [12]. The ICA-based hyperspectral crop classification methodologies assure the extracted feature is independent; nonetheless, ICA induces high computation overhead and doesn't guarantee to retain spatial information. On the other side, PCA-based hyperspectral crop classification methodologies realize good classification accuracy when compared with ICA- based methodologies. The PCA- based methodologies aid in assuring stabilizing features with a limited size of high meaningful representation. Nonetheless, PCA-based HSI crop classification methodologies are not efficient in retaining useful spectral features. Thus, for retaining spectral features more efficiently Image fusion (IF) methodologies are used in recent work. However, IF-based methodologies achieve poor classification performance; this is because they are affected due to the presence of noise and 
mixed pixel due to different illumination and climatic conditions [13], [14].

Recently, Deep Learning (DL) methodologies [15], [16] have been adopted for HSI crop classification [17], [18] with good accuracies [19], [20] which is studied in literature survey section. However, these DL-based methodologies induce high computation overhead and require a higher number of training parameters [21]. Further, induces high misclassification when data is imbalanced. For overcoming research problems it is important to extract meaningful features both spectrally as well as spatially; further, it is important to eliminate shading features from crop inherent features to classification accuracies. Here we used image fusion and recursive filter (IFRF) [22], [23] for obtaining semantic features across different bands i.e., spectrally. The usage of IFRF aided in reducing feature size with meaningful representation. Then we present an inherent feature extraction (IFE) method for distinguishing physical properties and shading elements of different crops. Existing models are trained using a Support vector machine (SVM) [18] for performing crop classification; the classification accuracies using SVM are affected due to misclassification [19]; especially when data is imbalanced and two objects exhibit similar physical features [20]. Thus, to address data imbalance issues and reduce misclassification in this we introduced a soft-margin decision boundary optimization model for SVM. The SMDBO-SVM based crop classification model aided in achieving high classification with less misclassification in comparison with the deep learningbased classification model.

The significance of using IFE-SMDBO-SVM is described below:

- Presented effective spatial-spectral feature extraction mechanism namely IFE. The IFE model can extract semantic features even under different illumination and climatic conditions.

- Presented soft-margin decision boundary optimization model for performing classification when HSI data exhibit data imbalance and also under mixed pixel environment.

- SMDBO-SVM based HSI achieves high classification accuracies with less misclassification (i.e., Kappa coefficient) in comparison with recent deep-learningbased HSI classification models.

- The SMDBO-SVM based HSI classification model reduces computation overhead in comparison with deep-learning-based HSI classification models.

The rest of the paper is organized as follows. Section II discusses various existing hyperspectral crop classification models and establishes the benefits and limitations, and hypotheses of the proposed method. Section III presents Inherent feature extraction and soft margin decision boundary optimization for Hyperspectral image-based crop classification methodology. In section IV, the performance efficiency of IFESMDBO in comparison with the existing HSI classification methodology is discussed. In the last section, the benefit of
IFE-SMDBO is discussed and the future direction of work is discussed.

\section{LITERATURE SURVEY}

This section presents some of the recent methodologies presented for performing crop classification using the hyperspectral image. In [2] presented HS-CNN (Hybrid Spectral Convolutional Neural Network) based HSI classification model. They first employed 3D-CNN for extracting spectral-spatial information followed by 2D-CNN. The classification performance of the HSCNN model heavily relies on both spectral and spatial information of HSI. The HSCNN model can joint retain spatial-spectral feature sets from different brands. The hybrid CNN model aids in learning more abstract level spatial features with minimal overhead in comparison with the 3D-CNN model. In [6], presented recurrent neural network (RNN)-based HSI classification. Here the spectral information is considered as a sequence; however, they showed standard RNN models are difficult to train and are not efficient as spatial features are not used. Thus, they presented Shorten Spatial-spectral RNN Parallel-GRU (St-SSpGRU) by combining convolution layers to achieve better HSI classification performance. In [16] showed that the 2D CNN just focused on extracting spatial features; however, neglects to extract spectral features. Similar, to [2], [21] presented a 3D CNN model that jointly considers extracting both spatial and spectral features; however, with reduced computational overhead by distributing spatial and spectral features extraction across different layers. In [15] showed CNN is widely used for HSI classification; However, they significantly because of high misclassification at the pixel level. In particular at the edges of neighboring crops; this is because the impact of adjacent pixels crops is different from target pixels. To address this, here they presented a center attention network (CAN) for HSI classification. The CAN-HSI can extract spatial and spectral features of both target pixel and adjacent pixels together in a simultaneous manner for performing HSI classification. In CAN major importance is given to highly correlated features concerning target pixels; thus aiding HSI classification performance. Further, CAM reduces parameters through a weighted sum of spatial and spectral features to reduce computation overhead without compromising on HSI classification accuracies.

In [14] showed DL-based method generally use patch-wise learning architecture for HSI classification. In recent times fast patch-free global learning (FPGA) frameworks have been modeled for HSI classification considering global spatial contextual information. Nonetheless, when HSI data is imbalanced the FPGA-based HSI classification finds it difficult in extracting discriminative features. To address they presented "spectral-spatial dependent global learning (SSDGL) architecture employing global joint attention (GJA) technique and global convolution LSTM (GCLSTM). In SSDGL for addressing data imbalance issues employed hierarchical tradeoffs sampling solution and weighted softmax loss function are modeled. The GCLSTM model is used for extracting LSTM dependencies of spectral features and later these dependencies are used for distinguishing spectral features for crop types. The GJA model is used for extracting attention areas for identifying the most discriminating features. In [17], 
for improving the robustness of standard machine learning models, recent work has emphasized integrating traditional ML models into DL methodologies. Here they studied modeling Deep SVM (DSVM) for HSI classification. The DSVM is modeled by implementing four kernel functions as polynomial, neural, Gaussian radial basis function, and exponential radial basis function. The standard SVM model is used for interconnecting weights of the entire network; the interconnecting weights act as a regularization parameter.

The research hypothesis the problems that existing SVMbased Hyperspectral object classification [18] are modeled using hard margin decision boundary [19], [20]; thus, high induce misclassification for smaller classes. Thus, are not efficient when data is imbalanced and induce high computational overheads. Further, the classification outcome is improved through better representation of spatial and spectral information; the proposed research work addresses the aforementioned problems in designing a better hyperspectral object classification model in the next section.

\section{INHERENT FEATURE EXTRACTION AND SOFT MARGIN DECISION BOUNDARY OPTIMIZATION FOR HYPERSPECTRAL IMAGE-BASED CROP CLASSIFICATION}

This work presents the inherent feature extraction (IFE) and Soft Margin Decision Boundary Optimization (SMDBO) Technique for Hyperspectral Crop Classification. Here first the working model of IFE-SMDBO based hyperspectral crop classification is presented. Second, present an inherent feature extraction model to reduce spectral features and exploit inherent features spatially to distinguish between actual crop and shadowing elements. Then, it discusses the standard SVM model used for HSI classification and highlights its limitation. Finally, present an improved decision boundary mechanism namely soft-margin decision boundary optimization (SMDBO) for addressing data imbalance and mixed pixel problems in HSI classification.

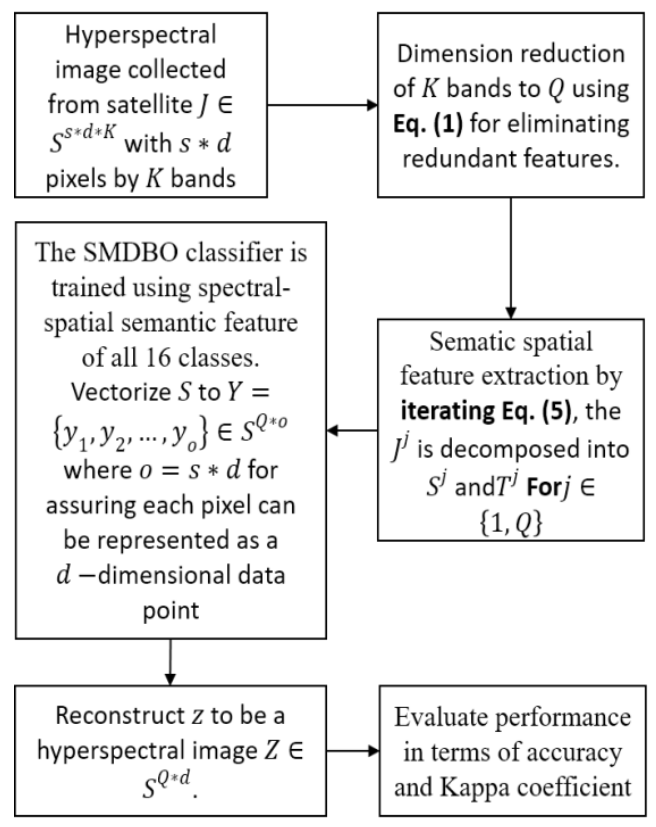

Fig. 1. Block Diagram of IFE-SMDBO based Hyperspectral Crop Classification Technique.

\section{A. Working Model of IFE-SMDBO based Hyperspectral Crop Classification}

The step involved in the proposed hyperspectral crop classification using the IFE-SMDBO model is shown in Fig. 1. The first step loads the HSI and reads the entire band information. Second, the HSI size is reduced spectrally using Eq. (1). Third, semantic features are extracted in an iterative manner using Eq. (5). Then, the sematic spatial-spectral feature bare trained using modified SMDBO and performs classification. Finally, the image is reconstructed for validating the accuracy of HSI classification models. The algorithm of the proposed IFE-SMDBO model is shown in Algorithm 1.

\section{Algorithm 1. IFE-SMDBO based hyperspectral crop classification technique.}

Input. Hyperspectral image collected from satellite $J \in S^{s * d * K}$ with $s * d$ pixels by $K$ bands.

Output. A classified outcome (i.e., labeled Hyperspectral image $Z$ ).

Step 1. Start.

Step 2. Dimension reduction of $K$ bands to $Q$ using Eq. (1).

Step 3. For $j \in\{1, Q\}$ do

Step 4. By iterating Eq. (5), the $J^{j}$ is decomposed into $S^{j}$ and $T^{j}$.

Step 5. End for.

Step 6.Vectorise $S$ to $Y=\left\{y_{1}, y_{2}, \ldots, y_{o}\right\} \in S^{Q * o}$ where $o=s * d$ for assuring each pixel can be represented as a $d$-dimensional data point.

Step 7. For obtaining labels $z \in S^{o}$ soft-margin decision boundary optimization-based support vector machine learning (SMDBO-SVM) algorithm is used.

Step 8. Reconstruct $z$ to be a hyperspectral image $Z \in$ $S^{Q * d}$.

\section{Step 9. Stop.}

\section{B. Band Selection and Feature Extraction}

Effective selection of band plays a very important role in achieving high accuracies with minimal computation overhead for performing crop classifications. Existing HSI classification methodologies used PCA for reducing band size; however, PCA fails to provide a higher number of useful features. Let consider hyperspectral data with $K$ bands which are reduced to $Q$ bands. Here we employ IFRF [23] for reducing band size and assuring eliminating noisy and redundant pixels spectrally through the following equation.

$J^{l}=\frac{\sum_{m=(l-1) n+1}^{l n} J^{m}}{n}, l=\left\lfloor\frac{K}{Q}\right\rfloor$,

where $n$ defines the sub-group band size considered, $l$ defines band indices of reduced spectral bands, $m$ defines band indices of actual spectral bands, and $[\cdot]$ a value closer to $-\infty$. 
Next, the inherent properties of different crops are extracted by eliminating shading elements in obtaining high-quality features spatially using the following equation.

$J_{q}=S_{q} T_{p}$

where $q$ defines pixel index, $T \in \mathcal{S}^{s * d}$ describes inherent features shading component, $S \in \mathcal{S}^{s * d}$ defining inherent feature component, and $J \in \mathcal{S}^{s * d}$ defines intensity feature. The variable $S_{q}$ and $T_{p}$ in the above equation are unknown; however, $J_{q}$ is a known variable. Generally, the reflectance value changes rapidly in edges and remains constant otherwise; similarly, the pixel with the same value will have the same reflectance value. Keeping the aforementioned context in consideration the $S_{q}$ is computed as follows

$S_{q}=\sum_{r \in \mathcal{O}(q)} b_{q r} S_{r}$,

wherer defines pixel index and $b_{q r}$ affinity matrix features for measuring similarities between $J_{q}$ and $J_{r}$.the adjacent pixel obtained through Gaussian window as follows

$G W=\exp \left(-\frac{\|q-r\|_{2}^{2}}{2 \sigma^{2}}\right)$

and $\sigma$ defines the size considered. Further, defining affinity graph (AG) plays a very essential part in semantically extracting inherent characteristics. Using Eq. (2) and Eq. (4), the meaningful feature is extracted through linear properties as follows

$\left\{\begin{array}{c}S_{r}=\sum_{r \in \mathcal{O}(q)} b_{q r} S_{r}, \\ \tilde{T}_{q}=\frac{1}{J_{q}} S_{r}\end{array}\right.$

where $\mathcal{O}(q)$ defines neighbor pixel $q, \widetilde{T}_{q}=\frac{1}{J_{q}}$ after obtaining the estimated value of $S_{r}$ and $T_{q}$. Thus each pixel physical properties of different crops are retained, where shading properties are not related to semantic feature sets properties and using inherent features the spatially useless feature can be eliminated.

\section{SVM Classification}

The feature space is represented as $Y \in S^{e}$, the index of different crops are represented as $Z=\{-1+1\}$, and respective crop distribution over $Y * Z$ is repressed as $E$. Let us consider that there are $o$ feature points in respective hyperspectral data and $n$ training features as described below

$T=\left\{\left(y_{1}, z_{1}\right),\left(y_{2}, z_{2}\right), \ldots,\left(y_{n}, z_{n}\right)\right\}$,

Here training features selected are identical based on the distribution of $E$. For predicting the sample considered the following function is defined

$f(y)=x^{U} \alpha(y)$,

where $x$ represent the forecaster, $\alpha(y)$ represent corresponding feature mapping of $y$ to kernel $L$ as described below

$L_{j k}=\alpha\left(y_{j}\right)^{U} \alpha\left(y_{k}\right)$.
In precise $Y$ represent the matrix where its $j^{\text {th }}$ column is $\alpha\left(y_{k}\right)$ which is defined as follows

$Y=\left[\alpha\left(y_{1}\right), \alpha\left(y_{2}\right), \ldots, \alpha\left(y_{n}\right)\right]$,

And $z$ is its column vector which is defined as follows

$y=\left(z_{1}, z_{2}, \ldots, z_{n}\right)^{U}$

The classification margin for describing a crop feature is computed using the following equation

$\beta_{j}=z_{j} x^{U} \alpha\left(y_{j}\right), j=1,2,3, \ldots, n$.

The state-of-art SVM based classification model generally considers that crop features are separable and the hyperplane has the capability in distinguishing the training crop features $T$ with no errors; thus, the SVM classification margin is obtained using the following equation

$\min _{x} \frac{1}{2}\|x\|^{2}$ such that $z_{j} x^{U} \alpha\left(y_{j}\right) \geq 1, j=1,2,3, \ldots, n$,

That maximizes its minimum margin.

\section{Soft Margin Decision Boundary Optimization Model}

Using above Eq. (12) will lead to high misclassification when used for classifying crops under a mixed cropping environment, when crops exhibit similar features, and when data is imbalanced. Further, there exist scenarios where a very limited feature is available for some crops and the high number of features for other crops; leading to concept drift and data imbalance issues. Using a hard-margin-based SVM classification model defined in the above equation gives a very poor result. Thus, for addressing this paper introduce soft margin decision boundary optimization SVM (SMDBO-SVM) for classifying crops considering concept drift and data imbalance issues. The SMDBO-SVM model optimizes the margin/boundary by minimizing the margin difference and simultaneously maximizing the margin average. Using Eq. (11), the margin difference is computed as follows

$\hat{\beta}=\frac{1}{n^{2}} \sum_{j=1}^{n} \sum_{k=1}^{n}\left[z_{j} x^{U} \alpha\left(y_{j}\right)-z_{j} x^{U} \alpha\left(y_{j}\right)\right]^{2}$

$=\frac{2}{n^{2}}\left(n x^{U} Y Y^{U} x-x^{U} Y x x^{U} Y^{U} x\right)$.

similarly, the margin means is obtained as follows

$\bar{\beta}=\frac{1}{n} \sum_{j=1}^{n} z_{j} x^{U} \alpha\left(y_{j}\right)=\frac{1}{n}(Y z)^{U} x$,

The Eq. (2) decision boundary can be optimized using the following equation

$\min _{x} \frac{1}{2}\|x\|^{2}+\delta_{1} \hat{\beta}-\delta_{2} \bar{\beta}$ such that $\alpha\left(y_{j}\right) \geq 1, j=$ $1,2,3, \ldots, n$

where $\delta_{1}$ and $\delta_{2}$ are parameters used for bringing good tradeoffs.

In non-distinguishable scenarios, the training crop features $T$ can't be distinguished with zero error and ideal hyperplane can't be obtained by minimizing objective function (tradeoffs model of error minimization and margin maximization). For addressing in SMDBO-SVM the error minimization are an 
additional parameter used for penalizing misclassified crop features, which is described below

$\min _{x, \mu} \frac{1}{2}\|x\|^{2} \delta_{1} \hat{\beta}-\delta_{2} \bar{\beta}+D \sum_{j=1}^{n} \mu_{j}$ such that $z_{j} x^{U} \alpha\left(y_{j}\right) \geq$ $1-\mu_{j}, \mu_{j} \geq 0, j=1,2,3, \ldots, n$,

where $\mu$ represent the slack parameter for quantitating feature loss and is computed defined as follows

$\mu=\left[\mu_{1}, \mu_{2}, \ldots, \mu_{n}\right]^{U}$

$D$ represent the regularization variable that is used for controlling the penalty given to misclassifications. The higher the error, the higher penalty is given to it.

Using Eq. (13) and Eq. (14) into Eq. (16) will result in quadratic programming problem as follows

$\min _{x, \mu} \frac{1}{2} x^{U} x+\frac{2 \delta_{1}}{n^{2}}\left(n x^{U} Y Y^{U} x-x^{U} Y z z^{U} Y^{U} x\right)-$

$\delta_{2} \frac{1}{n}(Y z)^{U} x+D \sum_{j=1}^{n} \mu_{j}$ such that $z_{j} x^{U} \alpha\left(y_{j}\right) \geq 1-\mu_{j}$,

$\mu_{j} \geq 0, j=1,2,3, \ldots, n$.

The ideal forecasting/prediction model $x^{*}$ for the optimization problem of Eq. (18) is defined using the following equation

$W^{*}=\sum_{j=1}^{n} \varphi_{j} \alpha\left(y_{j}\right)=Y \varphi$,

where $\varphi$ described its coefficient which is described as follows

$\varphi=\left[\varphi_{1}, \varphi_{2}, \varphi_{3}, \ldots \varphi_{n}\right]$.

Using Eq. (19) into Eq. (18), Eq. (18) is updated as follows $\min _{\varphi, \mu} \frac{1}{2} \varphi^{U} R \varphi+q^{U}+D \sum_{j=1}^{n} \mu_{j}$ such that $z_{j} \varphi^{U} L_{j} \geq 1-\mu_{j}$, $\mu_{j} \geq 0, j=1,2,3, \ldots, n$,

where the parameter $R$ is computed as follows

$R=4 \delta_{1} \frac{\left[n L^{U} L-(L z)(L z)^{U}\right]}{n^{2}+L}$,

Then, $q$ is computed as follows

$p=\frac{-\delta_{1} L z}{n}$,

The kernel matrix $L$ is computed as follows

$L=Y^{U} Y$,

and $L_{j}$ represents the $j^{\text {th }}$ column of kernel matrix $L$.

In general, the state-of-art SVM model are used for binary classification purpose; however, in this, we propose SMDBOSVM as a multiclass classifier. Let consider $Z=$ $\{1,2,3, \ldots, m\}$ as a set of crop classes in hyperspectral data; then, $m(m-1 / 2)$ hyperplane is built all probable pairwise classifier using SMDBO-SVM. Here the SMDBO-SVM model first performs binary classification among two classes $j$ and $k$ through discriminant function $f_{j k}(y) \in\{-1,1\}$ where $j \neq k$ and belongs to $Z$. Further, it is important for computing weighted function $T_{j}\left(y_{q}\right)$ for respective individual class $j \in Z$, before making any decision of predicted value $y_{q}$. Thus, a weighted strategy is modeled for distinguishing different crops from one another is described as follows

$T_{j}\left(y_{q}\right)=\sum_{\substack{k=1 \\ k \neq j}}^{m} \operatorname{sign}\left\{g_{j k}\left(y_{q}\right)\right\}$,

where $\operatorname{sign}(\cdot)$ represents the sign function used for binary representation of value. The decision of classified crop $y_{q}$ is done based on the highest weighted crops as described below

$j^{*}=\arg \max _{j \in Z}\left\{T_{j}\left(y_{q}\right)\right\}$.

The semantic feature extracted from HSI data is trained using the SMDBO-SVM model to aid in attaining better crop classification performance in comparison with the state-of-art crop classification model which is experimentally proven below.

\section{Simulation Analysis And Results}

This section evaluated the effectiveness of IFE-SMDBOSVM based hyperspectral crop classification over various recent state-of-art hyperspectral classification model [1], [4], [6], [10], [14], [15], and [17]. Total two publically available benchmarks HSI datasets such as Indian pines and Pavia University are used for analyzing HSI classification models. The performance of different classification models is measured using the most widely used metric in many existing HSI classification models such as average accuracy, overall accuracy, Kappa coefficient, and computation time. Attaining a higher accuracy value of accuracy and higher value of Kappa coefficient indicated good performance. Alongside, reducing time indicates the model is suitable for real-time deployment.

TABLE I. ThE GROUND TRUth DATA OF THE INDIAN Pines Dataset WITH 16 CLASSES

\begin{tabular}{|l|c|l|}
\hline Number & Classes & Total Samples \\
\hline $\mathbf{1}$ & Alfalfa & 46 \\
\hline $\mathbf{2}$ & Buildings Grass Trees Drives & 386 \\
\hline $\mathbf{3}$ & Corn notill & 1428 \\
\hline $\mathbf{4}$ & Corn mintill & 830 \\
\hline $\mathbf{5}$ & Corn & 237 \\
\hline $\mathbf{6}$ & Grass pasture & 483 \\
\hline $\mathbf{7}$ & Grass trees & 730 \\
\hline $\mathbf{8}$ & Grass pasture moved & 28 \\
\hline $\mathbf{9}$ & Hay windrowed & 478 \\
\hline $\mathbf{1 0}$ & Oats & 20 \\
\hline $\mathbf{1 1}$ & Soybean notill & 972 \\
\hline $\mathbf{1 2}$ & Soybean mintill & 2455 \\
\hline $\mathbf{1 3}$ & Soybean clean & 593 \\
\hline $\mathbf{1 4}$ & Stone Steel Towers & 93 \\
\hline $\mathbf{1 5}$ & wheat & 205 \\
\hline $\mathbf{1 6}$ & woods & 1265 \\
\hline & & \\
\hline
\end{tabular}




\section{A. Dataset Description}

The Indian Pines dataset is collected through an AVIRIS sensor deployed over the northern-west side of Indiana. The hyperspectral data is collected by setting a wavelength of $0: 4-2: 5 \times 10^{-6}$ meters with 224 bands and $145 \times 145$ pixels. The reason for using IP is because the majority of the area covered is the agriculture environment i.e., $2 / 3^{\text {rd }}$ and the remaining $1 / 3^{\text {rd }}$ measured areas are forest and other vegetation that is grown naturally. Further, IP data encompasses small roads, houses, low-lying buildings, and two-lane highways. Alongside, there are crops with early stages of growth which is less than $5 \%$ of overall data collected in IP. The ground truth data is composed of a total of 16 crops (i.e., labels) as shown in Table I. Similar, to [14]-[17], the water absorption bands are eliminated and spectral bands size are reduced to 200 .

The Pavia University hyperspectral data is collected through the ROSIS sensor. The PU dataset is measured with a spatial resolution of 1: 3 meters, with total 103 spectral bands, and composed of $610 \times 610$ pixels. Before analysis some data are eliminated they don't provide any information similar to [14]-[17]. The ground truth data is composed of total 9 classes as shown in Table II.

TABLE II. THE GRound TRUth DATA of PAVIA UnIVERsity DATASET WITH 9 CLASSES

\begin{tabular}{|l|l|l|}
\hline Number & Classes & Total Samples \\
\hline $\mathbf{1}$ & Asphalt & 6631 \\
\hline $\mathbf{2}$ & Bitumen & 1330 \\
\hline $\mathbf{3}$ & Bare - S & 5029 \\
\hline $\mathbf{4}$ & Gravel & 2099 \\
\hline $\mathbf{5}$ & Meadows & 18649 \\
\hline $\mathbf{6}$ & Painted - M - S & 1345 \\
\hline $\mathbf{7}$ & Shadow & 947 \\
\hline $\mathbf{8}$ & Sum & 42776 \\
\hline $\mathbf{9}$ & Self - B - B & 3682 \\
\hline
\end{tabular}

\section{B. Comparative Analysis for IndianPines Dataset}

Here experiment is conducted using Indian Pines Dataset for validating the performance achieved using IFE-SMDBOSVM and other state-of-art HSI crop classification methods such as CNN-AL-MRF, CAM, FPGA, SSDGL, and DSVM. The accuracies achieved for different classes of an object by the individual model are shown in Table III. From the experiment, it can be seen the proposed IFE-SMDBO-SVM achieves much better results than other HSI crop classification methods such as CNN-AL-MRF, CAM, FPGA, SSDGL, and DSVM in terms of accuracies and Kappa coefficient. Further, the IFE-SMDBO-SVM induces very little computation overhead in comparison with CNN-AL-MNF.
TABLE III. COMPARATIVE ANALYSIS OF IFE-SMDBO-SVM OVER RECENT HSI CROP CLASSIFICATION METHODOLOGY FOR INDIAN PINES DATASET

\begin{tabular}{|c|c|c|c|c|c|c|}
\hline $\begin{array}{l}\text { Class } \\
\text { name }\end{array}$ & $\begin{array}{l}\text { CNN- } \\
\text { Al- } \\
\text { MNF } \\
(2020) \\
{[10]}\end{array}$ & $\begin{array}{l}\text { CAM } \\
(2021) \\
{[15]}\end{array}$ & $\begin{array}{l}\text { FPGA } \\
(2020) \\
{[14]}\end{array}$ & $\begin{array}{l}\text { SSDGL } \\
(2021) \\
{[14]}\end{array}$ & $\begin{array}{l}\text { DSVM } \\
(2020) \\
{[17]}\end{array}$ & $\begin{array}{l}\text { IFE- } \\
\text { SMDBO- } \\
\text { SVM }\end{array}$ \\
\hline Alfalfa & 92.71 & 87.8 & 97.22 & 100 & 100 & 100 \\
\hline Corn notill & 92.98 & 98.05 & 93.07 & 99.63 & 100 & 99.98 \\
\hline $\begin{array}{l}\text { Corn } \\
\text { mintill }\end{array}$ & 88.7 & 97.99 & 89.46 & 99.24 & 100 & 99.97 \\
\hline Corn & 97.7 & 94.37 & 100 & 100 & 100 & 100 \\
\hline $\begin{array}{l}\text { Grass } \\
\text { pasture }\end{array}$ & 92.9 & 98.39 & 95.63 & 99.56 & 99.43 & 99.56 \\
\hline Grass trees & 98.89 & 99.7 & 97.56 & 100 & 98.89 & 99.88 \\
\hline $\begin{array}{l}\text { Grass } \\
\text { pasture } \\
\text { moved }\end{array}$ & 76.74 & 100 & 100 & 100 & 100 & 100 \\
\hline $\begin{array}{l}\text { Hay } \\
\text { windrowe } \\
\text { d }\end{array}$ & 97.87 & 100 & 100 & 100 & 98.72 & 100 \\
\hline Oats & 38.89 & 77.78 & 100 & 100 & 100 & 99.97 \\
\hline $\begin{array}{l}\text { Soybean } \\
\text { notill }\end{array}$ & 92.27 & 98.17 & 96.64 & 99.68 & 95.75 & 99.41 \\
\hline $\begin{array}{l}\text { Soybean } \\
\text { mintill }\end{array}$ & 95.07 & 98.33 & 96.74 & 99.36 & 100 & 99.46 \\
\hline $\begin{array}{l}\text { Soybean } \\
\text { clean }\end{array}$ & 90.51 & 97.94 & 91.65 & 99.11 & 99.63 & 100 \\
\hline wheat & 96.53 & 100 & 100 & 100 & 100 & 99.85 \\
\hline woods & 99.28 & 98.77 & 99.91 & 100 & 100 & 100 \\
\hline $\begin{array}{l}\text { Buildings } \\
\text { Grass } \\
\text { Trees } \\
\text { Drives }\end{array}$ & 88.4 & 92.51 & 99.72 & 100 & 95.45 & 99.87 \\
\hline $\begin{array}{l}\text { Stone } \\
\text { Steel } \\
\text { Towers }\end{array}$ & 97.12 & 98.81 & 100 & 100 & 100 & 100 \\
\hline OA (\%) & 98.79 & 98.1 & 96.18 & 99.63 & 98.86 & 99.7 \\
\hline AA (\%) & 94.28 & 96.16 & 97.33 & 99.79 & 99.24 & 99.87 \\
\hline $\begin{array}{l}\text { Kappa } \\
(\%)\end{array}$ & - & 97.84 & 95.64 & 99.58 & - & 99.66 \\
\hline Time (s) & $\begin{array}{l}8109.3 \\
4\end{array}$ & - & - & - & - & 12.5 \\
\hline
\end{tabular}




\section{Comparative Analysis for Pavia University Dataset}

Here experiment is conducted using Pavia University dataset for validating the performance achieved using IFESMDBO-SVM and other state-of-art HSI crop classification methods such as CNN-AL-MRF, CAM, FPGA, SSDGL, and DSVM. The accuracies achieved for different classes of an object by the individual model are shown in Table IV. From the experiment, it can be seen the proposed IFE-SMDBO-SVM achieves much better results than other HSI crop classification methods such as CNN-AL-MRF, CAM, FPGA, SSDGL, and DSVM in terms of accuracies and Kappa coefficient. Further, the IFE-SMDBO-SVM induces very little computation overhead in comparison with CNN-AL-MNF.

TABLE IV COMPARATIVE ANALYSIS OF IFE-DMDBO-SVM OVER RECENT HSI CROP CLASSIFICATION METHODOLOGY FOR PAVIA UNIVERSITY DATASET

\begin{tabular}{|c|c|c|c|c|c|c|}
\hline $\begin{array}{l}\text { Class } \\
\text { name }\end{array}$ & $\begin{array}{l}\text { CNN- } \\
\text { Al- } \\
\text { MNF } \\
(2020) \\
{[10]}\end{array}$ & $\begin{array}{l}\text { CAM } \\
(2021) \\
{[15]}\end{array}$ & $\begin{array}{l}\text { FPGA } \\
(2020) \\
{[14]}\end{array}$ & $\begin{array}{l}\text { SSDGL } \\
(2021) \\
{[14]}\end{array}$ & $\begin{array}{l}\text { DSVM } \\
(2020) \\
{[17]}\end{array}$ & $\begin{array}{l}\text { IFE- } \\
\text { SMDBO- } \\
\text { SVM }\end{array}$ \\
\hline Asphalt & 82.88 & 99.54 & 97.83 & 100 & 99.55 & 99.96 \\
\hline Meadows & 100 & 99.78 & 99.95 & 100 & 99.36 & 100 \\
\hline Gravels & 98.32 & 93.15 & 91.28 & 100 & 99.43 & 100 \\
\hline Trees & 99.76 & 98.63 & 95.85 & 99.67 & 99.45 & 100 \\
\hline $\begin{array}{l}\text { Painted } \\
\text { metal } \\
\text { sheets }\end{array}$ & 99.85 & 100 & 100 & 100 & 95.64 & 99.97 \\
\hline Bare soil & 100 & 99.78 & 99.76 & 100 & 100 & 100 \\
\hline Bitumen & 98.83 & 98.08 & 99.73 & 100 & 97.66 & 100 \\
\hline $\begin{array}{l}\text { Self- } \\
\text { blocking } \\
\text { bricks }\end{array}$ & 100 & 96.06 & 98.05 & 99.92 & 98.92 & 100 \\
\hline Shadows & - & 99.89 & 97.86 & 100 & 99.11 & 99.92 \\
\hline OA (\%) & 99.15 & 98.97 & 98.68 & 99.97 & 98.17 & 99.98 \\
\hline AA (\%) & 97.45 & 98.32 & 97.82 & 99.95 & 98.79 & 99.98 \\
\hline $\begin{array}{l}\text { Kappa } \\
(\%)\end{array}$ & - & 98.64 & 98.25 & 99.96 & - & 99.98 \\
\hline Time (s) & 1378.57 & - & - & - & - & 8.5 \\
\hline
\end{tabular}

\section{Accuracy performance for different training size}

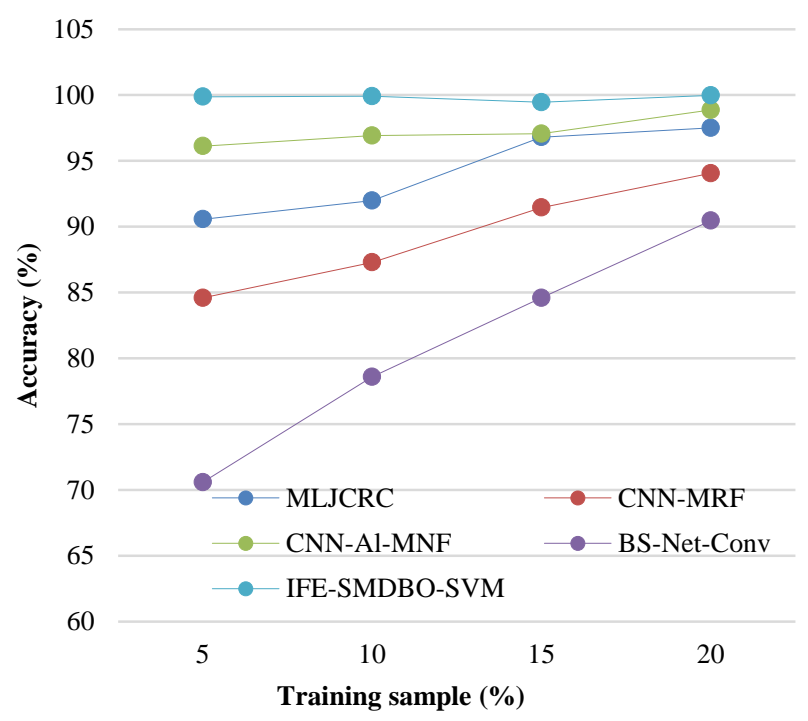

Fig. 2. The Classification Outcome was attained using IFE-SMDBO-SVM and Various Existing Classification Methods.

\section{Effect of Varying Training Sample size}

This section presents a comparative analysis of the proposed IFE-SMDBO-SVM classification over state-of-art HSI classification methodologies considering the effect training sample size. Here the training sample size is varied from 5 to $20 \%$ and the experiment is conducted as shown in Fig. 2. The existing HSI classification methods are MLJRC (Yang et al., 2018) [1], BS-Net-Conv (Cai et al., 2020) [7], and CNN-MBF (Cao et al., 2018) [4], and CNN-Al-MNF (Cao et al., 2020) [10]. From the result achieved it can be seen that among deep learning methodologies CNN-Al-MNF achieves very good performance with accuracies of $96.12 \%$ and $99.41 \%$ considering the training sample size of $5 \%$ and $20 \%$, respectively. On the other, BS-Net-Conv achieves very poor performance with accuracies of $70.58 \%$ and $90.45 \%$ considering the training sample size of $5 \%$ and $20 \%$, respectively. The IFE-SMDBO-SVM achieves very good performance accuracies with accuracies of $98.7 \%$ and $99.97 \%$ considering the training sample size of $5 \%$ and $20 \%$, respectively when compared with other state-of-art HSI classification algorithms such as MLJRC, BS-Net-FC, BS-NetConv, and CNN-MBF. From the result, the SFR-HSI is very efficient when there is a very limited training sample available.

\section{E. Effect of Inherent Feature Extraction Method}

This section evaluates the effect of using IFE in a classification task. The classification accuracies obtained by IFE-HSI and other existing HSI crop classification methodologies are graphically shown in Fig. 3. The effect of using and not using IFE is shown in Fig. 4. From Figure, it can be seen how IFE aids the classification accuracies enhancement. Thus, it can be stated the IFE-SMDBO-SVM model can learn crop inherent features more efficiently by eliminating the shadow component. 


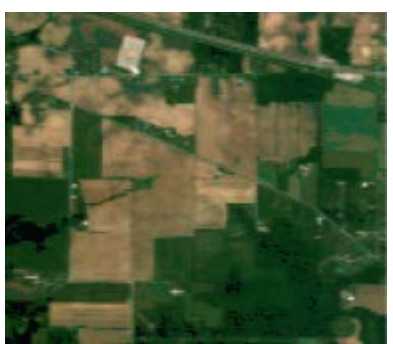

False Color Map

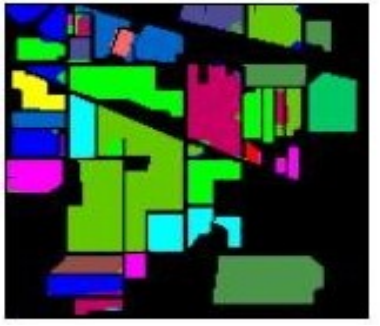

SVM-3DDWT-GC (94.28\%) [6]

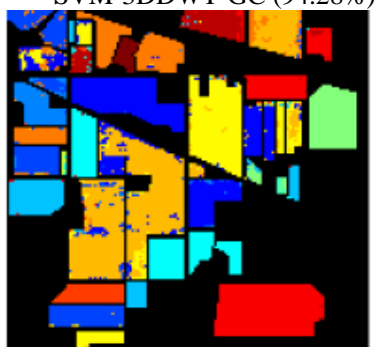

St-SS-GRU (86.28\%) [6]

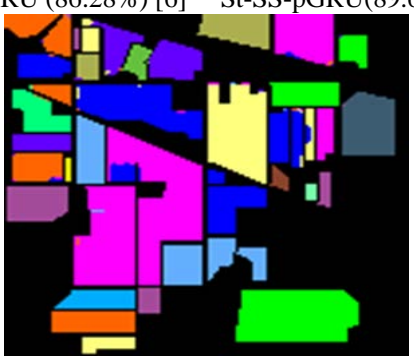

IFE-SMDBO-SVM (99.89\%)

Fig. 3. Classification Maps were Obtained by All Methods on the Indian Pines Dataset (Overall Accuracies are reported in Parentheses).

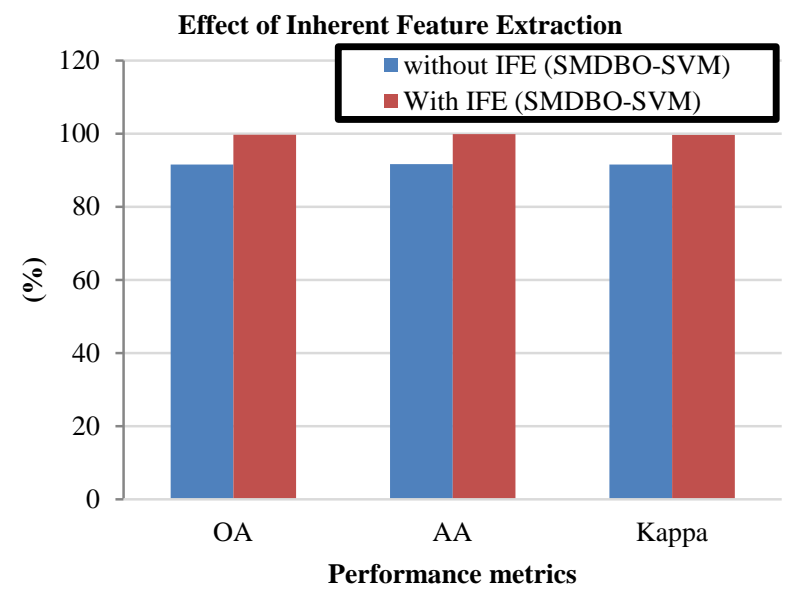

Fig. 4. Effect of IFE on Hyperspectral Object Classification Performance.

\section{CONCLUSION}

Designing hyperspectral crop classification with high accuracies with minimal computation time is challenging. In achieving high accuracies existing methodologies used machine and deep learning models; however, induces high training and computation overhead. In addressing computation overhead, dimension reduction technique has been emphasized; however, these model does not attain good accuracies due to poor spatial-spectral feature representation. This paper presented a hybrid design namely IFE-SMDBO-SVM using dimension reduction and machine learning model together for bringing tradeoffs among achieving higher accuracies with minimal time. The IFE-SMDBO-SVM works significantly well even with a fewer number of training samples; further, modeling of soft margin decision boundary aid in addressing feature imbalance issues during classification. The IFESMDBO-SVM much better result accuracies, Kappa coefficient, and computation time in comparison with recent HSI classification models such as SS-pGRU, CNN-Al-MNF, CAM, FPGA, SSDGL, and DSVM. The proposed model attains a much superior OA performance of $98.89 \%$ which is better than and slightly better than CNN-Al-MNF 2020. However, the accuracies of existing HSI methodologies are highly dependent on a higher number of samples and induce high computation overhead. However, IFE-SMDBO-SVM can work efficiently even with a small number of training samples with high speed. Thus, the proposed IFE-SMDBO-SVM model is much efficient when compared with existing hyperspectral image classification. In the future would consider introducing artificial noise into the hyperspectral image to meet the realtime requirement of the agriculture environment and see how the proposed HSI classification model can perform.

REFERENCES

[1] J. Yang and J. Qian, "Hyperspectral Image Classification via Multiscale Joint Collaborative Representation With Locally Adaptive Dictionary," in IEEE Geoscience and Remote Sensing Letters, vol. 15, no. 1, pp. 112116, Jan. 2018, doi: 10.1109/LGRS.2017.2776113.

[2] S. K. Roy, G. Krishna, S. R. Dubey, and B. B. Chaudhuri, "HybridSN: Exploring 3-D-2-D CNN Feature Hierarchy for Hyperspectral Image Classification," in IEEE Geoscience and Remote Sensing Letters, vol. 17, no. 2, pp. 277-281, Feb. 2020, doi: 10.1109/LGRS.2019.2918719.

[3] A. Santara et al., "BASS Net: Band-Adaptive Spectral-Spatial Feature Learning Neural Network for Hyperspectral Image Classification," in IEEE Transactions on Geoscience and Remote Sensing, vol. 55, no. 9, pp. 5293-5301, Sept. 2017, doi: 10.1109/TGRS.2017.2705073.

[4] X. Cao, F. Zhou, L. Xu, D. Meng, Z. Xu and J. Paisley, "Hyperspectral Image Classification With Markov Random Fields and a Convolutional Neural Network," in IEEE Transactions on Image Processing, vol. 27, no. 5, pp. 2354-2367, May 2018, doi: 10.1109/TIP.2018.2799324.

[5] Y. Liang, X. Zhao, A. J. X. Guo and F. Zhu, "Hyperspectral Image Classification With Deep Metric Learning and Conditional Random Field," in IEEE Geoscience and Remote Sensing Letters, vol. 17, no. 6, pp. 1042-1046, June 2020, doi: 10.1109/LGRS.2019.2939356.

[6] Luo, Haowen. (2018). Shorten Spatial-spectral RNN with Parallel-GRU for Hyperspectral Image Classification.

[7] Y. Cai, X. Liu and Z. Cai, "BS-Nets: An End-to-End Framework for Band Selection of Hyperspectral Image," in IEEE Transactions on Geoscience and Remote Sensing, vol. 58, no. 3, pp. 1969-1984, March 2020, doi: 10.1109/TGRS.2019.2951433.

[8] C. Yu et al., "Hyperspectral Image Classification Method Based on CNN Architecture Embedding With Hashing Semantic Feature," in IEEE Journal of Selected Topics in Applied Earth Observations and 
Remote Sensing, vol. 12, no. 6, pp. 1866-1881, June 2019, doi: 10.1109/JSTARS.2019.2911987.

[9] Lin, L., Chen, C. \& Xu, T. Spatial-spectral hyperspectral image classification based on information measurement and CNN. J Wireless Com Network 2020, 59 (2020). https://doi.org/10.1186/s13638-02001666-9.

[10] X. Cao, J. Yao, Z. Xu and D. Meng, "Hyperspectral Image Classification With Convolutional Neural Network and Active Learning," in IEEE Transactions on Geoscience and Remote Sensing, vol. 58, no. 7, pp. 4604-4616, July 2020, doi: 10.1109/TGRS.2020.2964627.

[11] Alotaibi, B., Alotaibi, M. A Hybrid Deep ResNet and Inception Model for Hyperspectral Image Classification. PFG 88, 463-476 (2020). https://doi.org/10.1007/s41064-020-00124-x.

[12] Ye, M., Ji, C., Chen, H. et al. Residual deep PCA-based feature extraction for hyperspectral image classification. Neural Comput \& Applic 32, 14287-14300 (2020). https://doi.org/10.1007/s00521-01904503-3.

[13] Gerland, Xin Wang, Guoqiang Wang, "Hyperspectral Band Selection Based on Adaptive Neighborhood Grouping and Local Structure Correlation", Journal of Sensors, vol. 2021, Article ID 5530385, 21 pages, 2021. https://doi.org/10.1155/2021/5530385.

[14] Zhu, Qiqi \& Deng, Weihuan \& Zheng, Zhuo \& Zhong, Yanfei \& Guan, Qingfeng \& Lin, Weihua \& Zhang, Liangpei \& Li, Deren. (2021). A Spectral-Spatial-Dependent Global Learning Framework for Insufficient and Imbalanced Hyperspectral Image Classification.

[15] Z. Zhao, D. Hu, H. Wang and X. Yu, "Center Attention Network for Hyperspectral Image Classification," in IEEE Journal of Selected Topics in Applied Earth Observations and Remote Sensing, vol. 14, pp. 34153425, 2021, doi: 10.1109/JSTARS.2021.3065706.
[16] Ahmad, Muhammad \& Shabbir, Sidrah \& Raza, Rana Aamir \& Mazzara, Manuel \& Distefano, Salvatore \& Khan, Adil. (2021). Hyperspectral Image Classification: Artifacts of Dimension Reduction on Hybrid CNN.

[17] Okwuashi, Onuwa \& Ndehedehe, Christopher. (2020). Deep support vector machine for hyperspectral image classification. Pattern Recognition. 103. 107298. 10.1016/j.patcog.2020.107298.

[18] Kalaiarasi, G., Maheswari, S. Deep proximal support vector machine classifiers for hyperspectral images classification. Neural Comput \& Applic (2021). https://doi.org/10.1007/s00521-021-05965-0.

[19] Pathak, D.K. \& Kalita, Sanjib \& Bhattacharyya, Dhruba K. (2021). Hyperspectral Image Classification using Support Vector Machine: a Spectral Spatial Feature Based Approach. Evolutionary Intelligence. 10.1007/s12065-021-00591-0.

[20] Guo, Y., Yin, X., Zhao, X. et al. Hyperspectral image classification with SVM and guided filter. J Wireless Com Network 2019, 56 (2019). https://doi.org/10.1186/s13638-019-1346-z.

[21] Yang X, Zhang X, Ye Y, Lau RYK, Lu S, Li X, Huang X. Synergistic 2D/3D Convolutional Neural Network for Hyperspectral Image Classification. Remote Sensing. 2020; 12(12):2033. https://doi.org/10.3390/rs12122033.

[22] M.C. Girish Babu, M.C. Padma, "A Efficient Solution for Classification of Crops using Hyper Spectral Satellite Images,” International Journal of Innovative Technology and Exploring Engineering (IJITEE)', ISSN: 2278-3075 (Online), Volume-9 Issue-2, December 2019, Page No. 5204-5211, 2019.

[23] M. C. Girish Baabu, Padma M. C. Semantic feature extraction method for hyperspectral crop classification. Indonesian Journal of Electrical Engineering and Computer Science, Vol. 23, No. 1, July 2021, pp. 387 395, ISSN: 2502-4752, DOI: 10.11591/ijeecs.v23.i1.pp387-395. 